\title{
Computation of the equilibrium three-particle entropy for dense atomic fluids by molecular dynamics simulation
}

\author{
Luca Maffioli, Nathan Clisby, Federico Frascoli, and B. D. Todda) \\ Department of Mathematics, Swinburne University of Technology, PO Box 218, Hawthorn, Victoria 3122, \\ Australia
}

We have computed the two and three-particle contribution to the entropy of a Weeks-Chandler-Andersen fluid via molecular dynamics simulations. The three-particle correlation function and entropy were computed with a new method which simplified calculation. Results are qualitatively similar to Lennard-Jones systems. We observed a numerical instability in the three-particle contribution. This phenomenon has been previously detected when the traditional method is used, thus it is likely to be intrinsic in the computation. While the effect of statistical fluctuations can be removed through an extrapolation procedure, the discretization error due to finite bin size is more difficult to characterize. With a correct choice of the bin size, a good estimate of the three-particle entropy contribution can be achieved at any state, even close to the freezing point. We observed that, despite the fact that the magnitude of the three-particle contribution increases significantly compared to the two-particle contribution as freezing is approached, the error induced from overestimation of the excess entropy by the two and three-body terms exceeds that induced by approximating the excess entropy with the two body term alone.

\section{INTRODUCTION}

Gibbs' entropy for a system of $N$ particles in thermodynamic equilibrium at temperature $T$ is defined as $\frac{1}{}$

$$
S=-k_{B} \int f(\boldsymbol{\Gamma}) \ln f(\boldsymbol{\Gamma}) \mathrm{d} \boldsymbol{\Gamma}
$$

with $f(\boldsymbol{\Gamma})$ the probability density function of the system in the phase space point $\Gamma$ and $k_{B}$ the Boltzmann constant. There are several way to compute the entropy of a system via computer simulation, and a direct estimation of the quantity in Eq. (1) can be considered the most direct way to achieve it. This is, however, unfeasible for systems of more than a few particles, due to the high dimensionality of the density function $f$. Two methods have been found to compute the entropy of a system of interacting particles via computer simulations. The first ${ }^{2}$ is based on the perturbation of the inter-particle potential from a reference system, in which the entropy can be computed exactly (in the case of a fluid, this system is the perfect gas), to the system of interest. An alternative method is based on the Green's expansion 1 , in which the total entropy of a system of $N$ particles is expressed as a sum of $N$ terms

$$
S=S_{1}+S_{2}+\ldots+S_{N}
$$

with a generic term $S_{i}$ representing the contribution to the total entropy of the $i$-body correlation. In this work, we will use the second method, as we focus, in the first place, on the computation of the three-body correlation function, and secondly, on the analysis of the contribution of each term to the total entropy of a system.

Defining $f_{N}^{(i)}$ as the probability density function of a generic subset of $i$ particles (with $i \leq N$ ), each term $S_{i}$ can be expressed as

$$
S_{i}=-\frac{k_{B}}{i !} \int f_{N}^{(i)}(1, \ldots, i) \ln \left(\delta f_{N}^{(i)}(1, \ldots, i)\right) \mathrm{d} \boldsymbol{\Gamma}_{1} \ldots \mathrm{d} \boldsymbol{\Gamma}_{\boldsymbol{i}},
$$

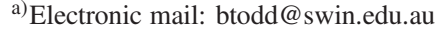

with

$$
\delta f_{N}^{(i)}(1, \ldots, i)=\frac{f_{N}^{(i)}(1, \ldots, i)}{\prod_{k=1}^{i} f_{N}^{(i-1)}(\{1, \ldots, i\} \backslash\{k\})} .
$$

Here the denominator in the second term of Eq. (3) represents the product of the correlation function of all possible subsets that can be formed removing one single element from the set $\{1, \ldots, i\}$.

In cases of low density the first two terms of the expansion $S_{1}$ and $S_{2}$, which can be computed straightforwardly via computer simulations, are able to detect up to $90 \%$ of the total entropy ${ }^{3}$.

Eq. (3) has been variously simplified, by first exploiting the fact that, for Canonical and Grand Canonical Ensembles, $f^{(i)}$ can be factorized into a product of momentum and configurational density functions ${ }^{4}$

$$
f_{N}^{(i)}=f_{N}^{(1)}\left(\mathbf{p}_{1}\right) \ldots f_{N}^{(1)}\left(\mathbf{p}_{i}\right) g_{N}^{(i)}\left(\mathbf{r}_{1}, \ldots, \mathbf{r}_{i}\right) .
$$

A local formulation (i.e. ensemble invariant) has been found ${ }^{5-7}$,

$$
\begin{aligned}
\frac{S}{N k_{B}} & =s_{p g}-\frac{\rho}{2} \int g^{(2)} \ln \left(g^{(2)}\right) d \mathbf{r}+\frac{\rho}{2} \int\left(g^{(2)}-1\right) d \mathbf{r} \\
& -\frac{\rho^{2}}{6} \int\left(g^{(3)} \ln \left(\delta g^{(3)}\right)\right) d \mathbf{r}^{2} \\
& +\frac{\rho^{2}}{6} \int\left(g^{(3)}-3 g^{(2)} g^{(2)}+3 g^{(2)}-1\right) d \mathbf{r}^{2}+\ldots
\end{aligned}
$$

Eq. (6) represents the first three terms of the local formulation of the Green's expansion. $\rho$ is the density of the system and $s_{p g}$ is the entropy of a perfect gas. It takes into account all information about momenta and about the configurational distribution function $g^{(N)}$ of a perfect gas in the same macroscopic state. We note that $s_{p g}$, whose expression is 7

$$
s_{p g}=\frac{5}{2}-\ln \left[\rho\left(\frac{h}{\left(2 \pi m k_{B} T\right)^{1 / 2}}\right)^{3}\right]
$$


with $m$ the mass of each particle, $h$ Planck's constant, does not correspond to $S_{1}$ in Eq. $3^{7}$; however such considerations are secondary for the purpose of this work. For the sake of clarity, the subscript $N$ will be omitted from now on.

Eq. (6) has been used to compute the entropy of a system of Lennard-Jones particles in various macroscopic conditions, and has been shown to be able to detect no less than $70 \%$ of the total entropy ${ }^{3}$. However, computation of $s_{3}$ is affected by a severe numerical instability, which manifests itself as an unphysical drift for large distances, and makes the estimate of the three-body entropy challenging. This behavior has been detected in previous works 3,8 , and correctly attributed to numerical issues, although the limited computational resources available at the time have not allowed a systematic investigation of it. Furthermore, the computation of the three-particle function $g^{(3)}$ is not trivial, and requires care.

In this work we first present a simpler method for the computation of the three-particle function. Secondly, the two and thee-particle entropy is computed for $\mathrm{WCA}^{9}$ particles, the causes of this drift are analyzed, and a method to correct it is presented.

\section{THE DIMENSIONLESS METHOD}

\section{A. The traditional method}

At equilibrium, two and three-body distribution functions, $g^{(2)}$ and $g^{(3)}$, depend respectively only on the distance between particles, $r, s, t$. With a small abuse of notation

$$
\begin{aligned}
g^{(2)}\left(\mathbf{r}_{1}, \mathbf{r}_{2}\right) & =g^{(2)}(r) \\
g^{(3)}\left(\mathbf{r}_{1}, \mathbf{r}_{2}, \mathbf{r}_{3}\right) & =g^{(3)}(r, s, t)
\end{aligned}
$$

and from Eq. (6), we have

$$
\begin{aligned}
s_{2}(R)= & -\frac{4 \pi \rho}{2} \int_{0}^{R}\left(g^{(2)}(r) \ln g^{(2)}(r)-g^{(2)}(r)+1\right) r^{2} \mathrm{~d} r \\
s_{3}(R)=- & \frac{8 \pi^{2} \rho^{2}}{6} \int_{0}^{R} \int_{0}^{R} \int_{0}^{R}[ \\
& g^{(3)}(r, s, t) \ln \left(\frac{g^{(3)}(r, s, t)}{g^{(2)}(r) g^{(2)}(s) g^{(2)}(t)}\right) \\
& -g^{(3)}(r, s, t)-g^{(2)}(r)-g^{(2)}(s)-g^{(2)}(t) \\
& +g^{(2)}(r) g^{(2)}(s)+g^{(2)}(r) g^{(2)}(t)+g^{(2)}(s) g^{(2)}(t) \\
& +1] r s t \mathrm{~d} r \mathrm{~d} s \mathrm{~d} t .
\end{aligned}
$$

As $R$ increases, the correlation between particles tends to zero and both $s_{2}$ and $s_{3}$ converge to their asymptotic values. Our goal is to estimate

$$
\begin{aligned}
& \lim _{R \rightarrow \infty} s_{2}(R) \\
& \lim _{R \rightarrow \infty} s_{3}(R) .
\end{aligned}
$$

Distribution functions are approximated with a histogram with bin size $\Delta$. During computer simulations, histograms are periodically updated by counting the number of realizations occurring in each bin, i.e. all pairs and triplets of particle distances which lay within each bin volume. Each value is then normalized by the distribution of a perfect gas in the same macroscopic condition 10 . These can be expressed as

$$
\begin{aligned}
g_{i}^{(2)} & =\frac{\left\langle N_{i}\right\rangle}{\rho N V_{i}^{(2)}} \\
g_{i, j, k}^{(3)} & =\frac{\left\langle N_{i, j, k}\right\rangle}{\rho^{2} N V_{i, j, k}^{(3)}}
\end{aligned}
$$

with

$$
\begin{aligned}
& V_{i}^{(2)}=\frac{4 \pi}{3} \int_{(i-1) \Delta}^{i \Delta} r^{2} \mathrm{~d} r \\
& V_{i, j, k}^{(3)}=8 \pi^{2} \int_{(i-1) \Delta}^{i \Delta} \int_{(j-1) \Delta}^{j \Delta} \int_{(k-1) \Delta}^{k \Delta} r s t \mathrm{~d} r \mathrm{~d} s \mathrm{~d} t .
\end{aligned}
$$

Due to the triangle inequality, the domain of the threeparticle distribution function is delimited by the planes

$$
\begin{aligned}
& r=s+t \\
& s=r+t \\
& t=r+s .
\end{aligned}
$$

Thus, bins that are crossing these borders have a smaller volume, as they are partially occupied by the domain. We call $R, S, T$ the integer coordinates of a generic bin, along the directions $r, s, t$ respectively. A generic triplet $R, S, T$ (with $R, S, T=1,2,3, \ldots)$ denotes the region $[(R-1) \Delta, R \Delta) \times[(s-$ 1) $\Delta, S \Delta) \times[(T-1) \Delta, T \Delta)$, and the triangle inequality implies that the three discrete indices satisfy

$$
\begin{gathered}
R \leq S+T \\
S \leq R+T \\
T \leq R+S .
\end{gathered}
$$

If we limit our calculation to the region $\left[0, R_{\max }\right) \times$ $\left[0, R_{\max }\right) \times\left[0, R_{\max }\right)$ the domain is symmetric by any permutation of indices, and we can focus our analysis on the region $R \geq S \geq T$, where the triangle inequality takes the form $T \geq R-S$. In this framework, any bin for which $T \geq R-S+2$ is fully immersed in the domain of the three particle function, and its volume is

$$
\begin{aligned}
V_{R, S, T}^{(3)} & =8 \pi^{2} \int_{(R-1) \Delta}^{R \Delta} \int_{(S-1) \Delta}^{S \Delta} \int_{(T-1) \Delta}^{T \Delta} r s t \mathrm{~d} r \mathrm{~d} s \mathrm{~d} t \\
& =8 \pi^{2}(R-1 / 2)(S-1 / 2)(T-1 / 2) \Delta^{6}
\end{aligned}
$$

The border bins $(R-S \leq T<R-S+2)$, exhibit a smaller volume. Particularly, we can distinguish the case where a bin is crossing one, two or three planes respectively:

- one plane: $T=R-S, T=R-S+1$, with $R \neq S, R \neq 1$, $S \neq 1$; 
- two planes: $T=1$, with $R=S$;

- three planes: $T=R=S=1$.

Each distinct case has to be computed separately, i.e. no general expression, valid for each possible case, can be found. Besides this, each volume must be further decomposed in smaller elementary volumes. As an example, the case of three plane intersections must be computed via 4 different integrals:

$$
\begin{aligned}
V_{1,1,1}=8 \pi^{2}\left[\int_{0}^{\Delta / 2} \int_{0}^{r} \int_{r-s}^{r+s} r s t \mathrm{~d} r \mathrm{~d} s \mathrm{~d} t\right. \\
+\int_{\Delta / 2}^{\Delta} \int_{0}^{\Delta-r} \int_{r-s}^{r+s} r s t \mathrm{~d} r \mathrm{~d} s \mathrm{~d} t \\
+\int_{\Delta / 2}^{\Delta} \int_{\Delta-r}^{\Delta / 2} \int_{r-s}^{\Delta} r s t \mathrm{~d} r \mathrm{~d} s \mathrm{~d} t \\
\left.+\int_{\Delta / 2}^{\Delta} \int_{\Delta / 2}^{r} \int_{r-s}^{\Delta} r s t \mathrm{~d} r \mathrm{~d} s \mathrm{~d} t\right]
\end{aligned}
$$

We do not show the explicit formula of each case, as it has been provided in a work of Baranyai and Evans ${ }^{3}$. It is clear, however, that this direct method is tricky, and we expect that it might be extremely challenging to extend this approach to the computation of higher order correlation functions, regardless of the other technical difficulties related to such a calculation.

\section{B. Proposed new dimensionless method}

We now show a more direct method to discretize the space of the three distances, such that no correction of border bins is required. Exploiting the symmetry of $g^{(3)}$ by any permutation of particles, e.g.

$$
g^{(3)}\left(\mathbf{r}_{1}, \mathbf{r}_{2}, \mathbf{r}_{3}\right)=g^{(3)}\left(\mathbf{r}_{3}, \mathbf{r}_{2}, \mathbf{r}_{1}\right) \Rightarrow g^{(3)}(r, s, t)=g^{(3)}(t, s, r)
$$

we can restrict the volume of interest to the region $r \geq s \geq t$, and combining it with the triangle inequality, we get

$$
r \in\left[0, R_{\max }\right], \quad s \in\left[\frac{r}{2}, r\right], \quad t \in[r-s, s]
$$

where $R_{\max }$ is the maximum particle separation distance chosen for the calculation. The three distances can now be transformed into dimensionless counterparts

$$
\begin{array}{ll}
r^{\prime}=\frac{r}{R_{\max }} & r^{\prime} \in[0,1] \\
s^{\prime}=\frac{2}{r}\left(s-\frac{r}{2}\right) & s^{\prime} \in[0,1] \\
t^{\prime}=\frac{t-r+s}{2 s-r} & t^{\prime} \in[0,1] .
\end{array}
$$

The new domain is trivially cubic (see Figure 1) and so perfectly fitted by a cubic bin grid. The elementary volume can

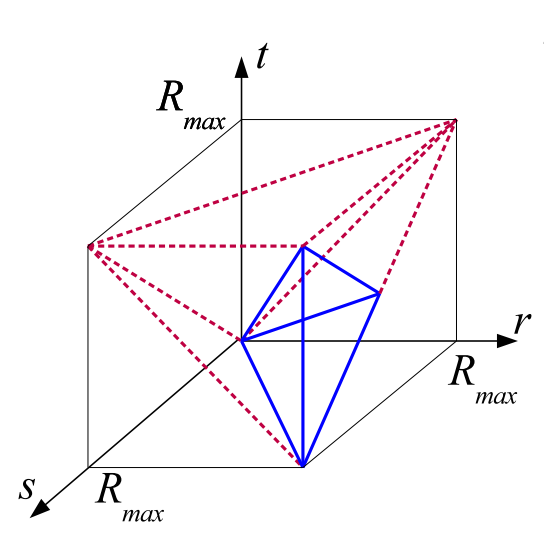

(a)
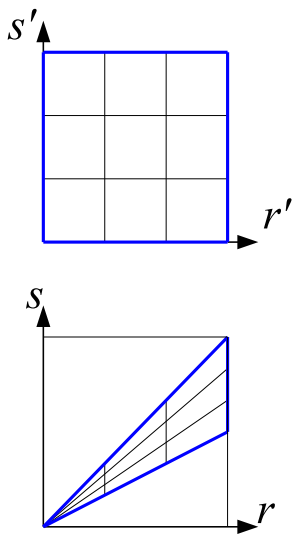

(b)
FIG. 1: (a): The three-particle function's domain (red borders, dotted), and the region chosen for the dimensionless method (blue borders, solid). (b) Cubic grid in the dimensionless space for the first two distances , $r^{\prime}, s^{\prime}$ and the corresponding grid in the original space.

be expressed by

$$
\begin{aligned}
d v(r, s, t) & =r s t \mathrm{~d} r \mathrm{~d} s \mathrm{~d} t= \\
& =\frac{R_{\text {max }}^{6}}{4}\left(r^{\prime}\right)^{5} s^{\prime}\left(s^{\prime}+1\right)\left(t^{\prime} s^{\prime}+\frac{1-s^{\prime}}{2}\right) \mathrm{d} r^{\prime} \mathrm{d} s^{\prime} \mathrm{d} t^{\prime}
\end{aligned}
$$

The three-particle correlation function, $g^{(3)}$, as well as $s_{3}$ can now be computed more straightforwardly. However, the main inconvenience of this method is the emergence of an unavoidable mismatch between the grid used for $g^{(2)}$ and the one used for $g^{(3)}$. In the computation of $s_{3}$, the two-particle distribution function is approximated as constant within each bin of the histogram of $g^{(3)}$, equal to its value in the barycentre of the bin.

In this work, we computed the two and three-particle entropy of Weeks-Chandler-Andersen (WCA) particles in different conditions. The WCA potential is defined as $\stackrel{9}{-}$

$$
\Phi_{W C A}(r)= \begin{cases}4 \varepsilon\left[\left(\frac{\sigma}{r}\right)^{12}-\left(\frac{\sigma}{r}\right)^{6}\right]+\varepsilon & \text { if } r \leq 2^{\frac{1}{6}} \sigma \\ 0 & \text { if } r>2^{\frac{1}{6}} \sigma\end{cases}
$$

with $r$ the inter-particle distance, $\sigma$ the diameter of the particles and $\varepsilon$ the potential well depth. We selected a case of low density (0.3), intermediate (0.7) and high density (0.92), at reduced temperature of $T=1.15$, and various states close to the freezing line $\frac{11,12}{}(\rho=0.92, T=0.75 ; \rho=1, T=1.5$; $\rho=1, T=2$ ). We analyzed the numerical instability of the three-particle entropy and we compared the standard method and the new dimensionless one. 


\section{COMPUTATIONAL DETAILS}

We performed molecular dynamics simulations for systems of 6750 WCA particles with diameter $\sigma$ and mass $m$ at different states. The system size, $L$, was varied based on the density from a minimum of $L=19.43 \sigma$ to a maximum of $L=28.31 \sigma$. All variables from now on are provided in reduced form, and $\sigma=1, \varepsilon=1, m=1$. We used a fourth order Gear predictor-corrector scheme ${ }^{13}$ to integrate the equations of motion, with reduced time step $\Delta t=0.001$. All systems were thermostatted with a Gaussian isokinetic thermostat. Periodically, all pairs and triplets of particles which laid within a distance of $R_{\max }=8.6362 \sigma$ from each other were used to update the two and three-particle histograms. The frequency of the sampling was chosen such that the total simulation time was equally distributed in the three operations consisting of, respectively, updating positions and momenta via the integration of the equations of motion, updating $g^{(2)}$, and updating $g^{(3)}$. Since a single update of $g^{(2)}$ is computationally faster than an update of $g^{(3)}$, the frequency of the two updates was different. The number of time steps between two updates was about 70 for the two-particle function and $10^{5}$ for the threeparticle function. These values are however merely representative, as they were affected by the density of the system.

Particles were started in a cubic lattice, and the momenta were randomly generated and rescaled in order to have zero net momentum along each direction and the desired initial temperature. An initial warm-up of $10^{5}$ time steps was used to allow the system to relax before commencing the sampling of the distribution functions. A uniform grid in the dimensionless space entails that the grid gets coarser as the separation distance between particles increases (cf. Figure 1). In order to guarantee a good match between the two and three-particle grid, the two-particle function was discretized with a much finer grid than $g^{(3)}$. The three-particle histogram was created using a grid of 300 bins per side. As a reference, we use the bin size along the first distance, $r^{\prime}$, as it is only rescaled by a factor of $R_{\max }$ (cf. Eq. (18). In the real space, bin size $\Delta_{b}$ is $R_{\max } / 300 \simeq 2.88 \times 10^{-2} \sigma$. The two-particle function was discretized with a grid of $3 \times 10^{6}$ bins and thus with a bin size of $\Delta=10^{-4} \Delta_{b}$. Each $g^{(3)}$ was obtained via $\simeq 850$ samples, for a total amount of $\simeq 2.7 \times 10^{12}$ triplets sampled from each run, resulting in an average number of realizations per bin $\bar{N}_{b} \simeq 10^{5}$. About $7.5 \times 10^{5}$ samples and $\simeq 1.2 \times 10^{13}$ total pairs were sampled for $g^{(2)}$, with an average number of realizations per bin of $\simeq 4 \times 10^{6}$.

The selection of the triplets of particles requires some consideration. For a generic particle $i$, all neighbor particles, i.e. closer than $R_{\max }$, are identified. This procedure guarantees that, given two neighbors $j, k$, the inter-particle distances $r_{i j}$ $r_{i k}, r_{j k}$ are in the following ranges

$$
\begin{aligned}
& 0 \leq r_{i j}<R_{\max } \\
& 0 \leq r_{i k}<R_{\max } \\
& 0 \leq r_{j k}<2 R_{\max }
\end{aligned}
$$

The constraint of avoiding self interaction requires every distance to be smaller than $L$. On the other hand, the presence of any distance in the range $[L / 2, L)$ entails that more than one set of distances may be found for the same triplet of particles, due to interaction with particle images. It is usually preferred to set $R_{\max }=L / 4$ and avoid any possible multiple interaction ${ }^{14}$. In a previous work ${ }^{3}$ the two ranges $R_{\max }=L / 2$ and $R_{\max }=L / 4$ were tested, and no statistical difference was found between them. We note that with such a scheme, the distances are not symmetric by a permutation of particles. This gives rise to two further problems: the domain of $g^{(3)}$ is no longer cubic and symmetric, as depicted in Sec. ПB. and the calculation of the three distances must be performed for any permutation of $i, j, k$, with an increment of the number of calculations of 6 times. In this work, we preferred to set the range of each distance

$$
\begin{aligned}
& 0 \leq r_{i j}<R_{\max } \leq L / 2 \\
& 0 \leq r_{i k}<R_{\max } \leq L / 2 \\
& 0 \leq r_{j k}<R_{\text {max }} \leq L / 2 .
\end{aligned}
$$

This constraint entails some advantages:

- any permutation of $i, j, k$ locates the same triplet of distance, making the update of $g^{(3)} 6$ times faster, and allowing to reduce the domain in the way previously described, making the histogram of $g^{(3)} 6$ times smaller.

- $R_{\text {max }}$ can be set up to $L / 2$ avoiding a too cautious constraint of $L / 4$.

Once a triplet of particles was obtained, the three distances were sorted and rescaled with the formula provided in Eq. (18), and the corresponding bin was updated.

The simulations were performed on the Swinburne supercomputer cluster OzSTAR, using multiple central processing unit (CPU) Intel Gold 6140 processors. On these CPUs, each simulation was about 156 hours long, each time step was performed in $\simeq 1.8 \times 10^{-3} \mathrm{~s}$, each update of $g^{(2)}$ required about $1.2 \times 10^{-1} \mathrm{~s}$, and each update of $g^{(3)}$ about $186 \mathrm{~s}$.

\section{RESULTS AND DISCUSSION}

Results in Figures 2,3 show the two and three-particle entropy computed at different conditions of density and temperature. The three-particle entropy is affected by the numerical drift. Previous analysis 3 ascribed the drift mainly to statistical fluctuations due to the small number of samples, and to a lesser extent, to the grid (i.e. bin size) used to histogram $g^{(3)}$. We found that, when the new dimensionless method is used, both the small number of samples and the finite bin size $\Delta$ have a key role in the promotion of the drift, as we will now discuss.

\section{A. Effect of statistical noise}

Numerical error induced by a small number of samples has a twofold aspect: due to the non linear expression of $s_{3}$, statistical fluctuation of $g^{(3)}$ around its true value results in both 


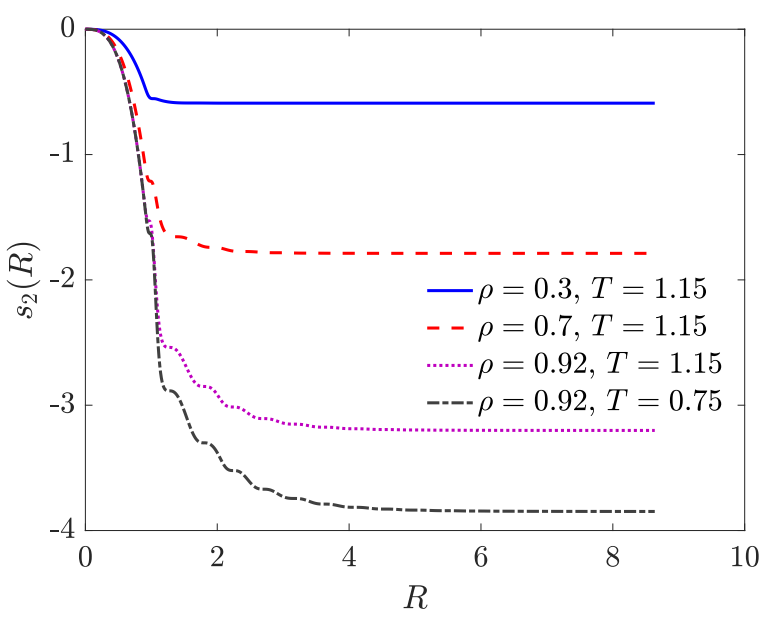

FIG. 2: Two-particle entropy $s_{2}$ for different conditions.

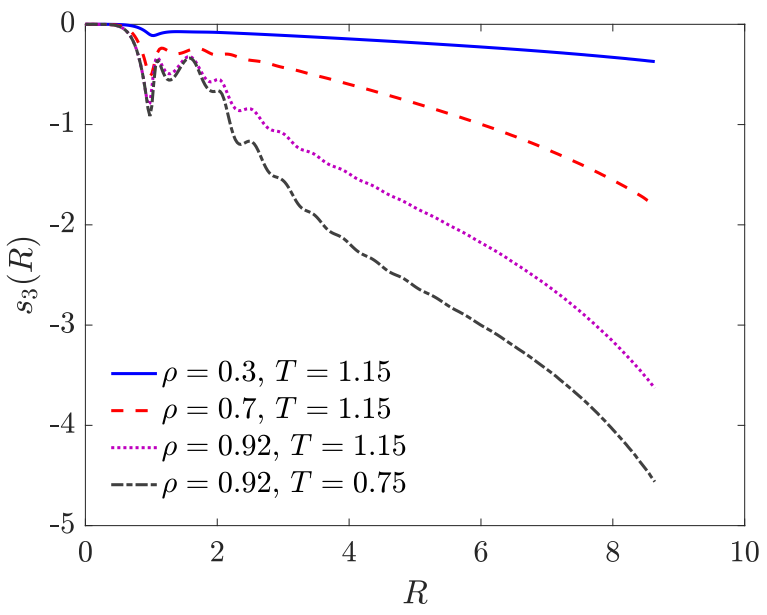

FIG. 3: Three-particle entropy $s_{3}$ for different conditions.

statistical and systematic error (i.e. drift) in the three-particle entropy. Increasing the number of samples reduces the error, however, an estimate of the residual drift and statistical fluctuations is necessary, as well as a method to extrapolate the distribution of the three-particle entropy in the infinite sample limit. Our goal is to generate the statistical distribution of the three-particle entropy for different levels of noise (i.e. equivalently, for different number of samples used to compute the two and three-particle correlation functions). A set of 48 two and three-particle distributions was obtained from the same number of independent simulations. Each distribution had the number of samples and bin size described in Sec. III] Merging together a certain number $M$ of these distribution functions allows us to increase the number of samples used to compute $s_{3}$, roughly of the order $M$. We define

$$
s_{3}(R)^{M}=s_{3}\left(\left\langle g^{(2)}\right\rangle_{M},\left\langle g^{(3)}\right\rangle_{M}\right)
$$

the statistical distribution of the entropy computed via $M$ two and three-particle functions merged together. We selected a
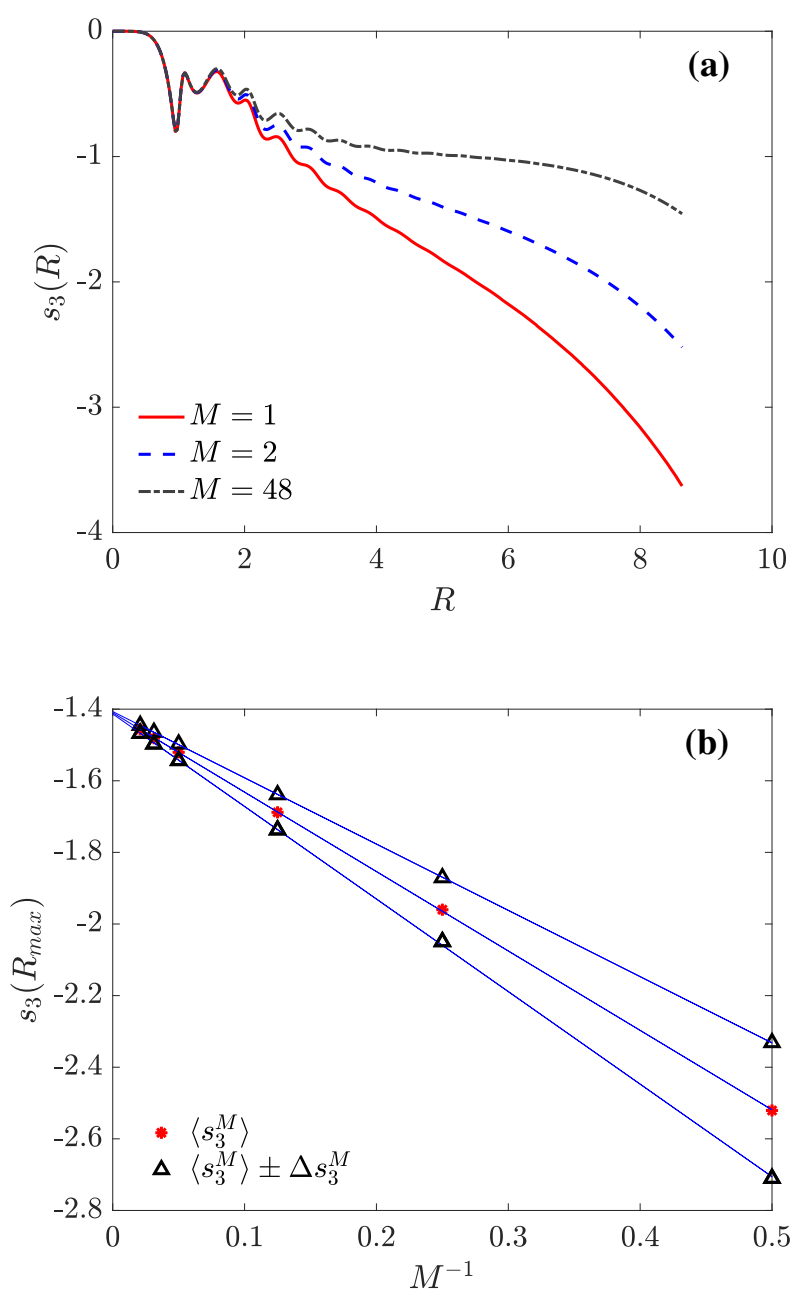

FIG. 4: (a) Magnitude of the drift for different numbers of samples. (b) Convergence of systematic effect (sample average, red points) and statistical fluctuations around the sample average, depicted via the standard deviation $\Delta s_{3}$ (black triangles), with respect to the number of samples. Blue lines represent the linear interpolation of both effects. State point $\rho=0.92, T=1.15$.

set of values $M(M=2,4,8,20,32,48)$ and for each value, the statistical distribution of $s_{3}$ was approximated via 200 different computation of $s_{3}$ : each $s_{3}$ was computed by merging $M$ two and three-particle functions, randomly extracted with repetitions from the original 48 distributions. Results for the state point $\rho=0.92, T=1.15$ are shown in Figure 4. It is clear that both the systematic and the statistical fluctuations converge with a law $\propto M^{-1} \propto N_{s}^{-1}$, with $N_{s}$ the number of samples used to generate $g^{(2)}$ and $g^{(3)}$. These results are in good agreement with previous works ${ }^{3}$.

This procedure allowed us to determine the law of the convergence of $s_{3}$ with respect to the statistical noise in $g^{(2)}$ and $g^{(3)}$. However, these data cannot be used to extrapolate the infinite sample limit, as they are highly correlated. The extrapolation would not differ substantially from the direct computation of $s_{3}$ when all 48 distributions are merged together 
without repetitions, and a linear drift is still well detectable even in the extrapolated function. In order to remove the bias induced by the inter-dependence of the data, a different procedure was used: with a proper choice of the values $M$, it is possible to generate a sequence of $s_{3}$ with different levels of noise in which each one of the original 48 distributions appears only once. A possible choice could be $M=1,2,4,8,33$. As we see, the sum of all $M$ is 48 , so no repeated distributions appear in the sequence. The 48 distributions are merged together with the rule previously described. In this procedure, one single $s_{3}$ is associated to each value of $M$. As an example, calling $g_{i}^{(n)}$ the $i$-th distribution function over the original 48 , the set $\left\{s_{3}{ }^{M}\right\}$ may be composed by 5 different $s_{3}$ computed in the following way:

$$
\begin{aligned}
s_{3}{ }^{1} & =s_{3}\left(g_{1}^{(n)}\right) \\
s_{3}{ }^{2} & =s_{3}\left(\left\langle g_{2,3}^{(n)}\right\rangle\right) \\
s_{3}{ }^{4} & =s_{3}\left(\left\langle g_{4, \ldots, 7}^{(n)}\right\rangle\right) \\
s_{3}{ }^{8} & =s_{3}\left(\left\langle g_{8, \ldots, 15}^{(n)}\right\rangle\right) \\
s_{3}{ }^{33} & =s_{3}\left(\left\langle g_{16, \ldots, 48}^{(n)}\right\rangle\right)
\end{aligned}
$$

This sequence was used to extrapolate the infinite sample limit with the law previously derived. The results are unbiased, as the data are independent, but they may be still affected by the order in which the 48 distributions appear in the sequence of Eq. (24). In order to remove this effect, we generated the statistical distribution of the extrapolated value via 600 different computations obtained permuting the order of the 48 distributions in Eq. (24). The sample average has then been selected as the estimate of $s_{3}$ in the infinite sample limit. Results are shown in Figures 5,6 It is evident that an extrapolation is necessary, particularly at low densities, as the small value of the true entropy and a smaller drift induced by the grid size makes the noise effect the primal source of error in the estimate of $s_{3}$. At higher densities, grid size effect is largely increased, and the noise has a secondary role in the drift. The standard deviation of the extrapolated $s_{3}$ is roughly constant for all different systems, and it is up to 0.02 at $R=R_{\max }$. At that distance, however, accuracy of the extrapolation is nullified by the grid size effect. For shorter distances it becomes almost negligible, being about 0.005 at $R=R_{\max } / 2$.

\section{B. Effect of the discretization}

At low and intermediate densities the grid size effect is essentially negligible, since $s_{3}$ reaches its asymptotic value well before the drift starts to be relevant. For $\rho=0.3$ and $T=1.15$ the convergence is very fast, and $s_{3}$ can be considered converged at $R=3 \sigma$; at intermediate densities, the inter-particle distance of convergence is about $4 \sigma$. In these cases, the grid size effect can be ignored, as $s_{3}$ exhibits a clear plateau with neither oscillations nor drift. At high densities, oscillations have not yet ceased when numerical instability starts to become relevant. A finer grid is able to reduce the magnitude of

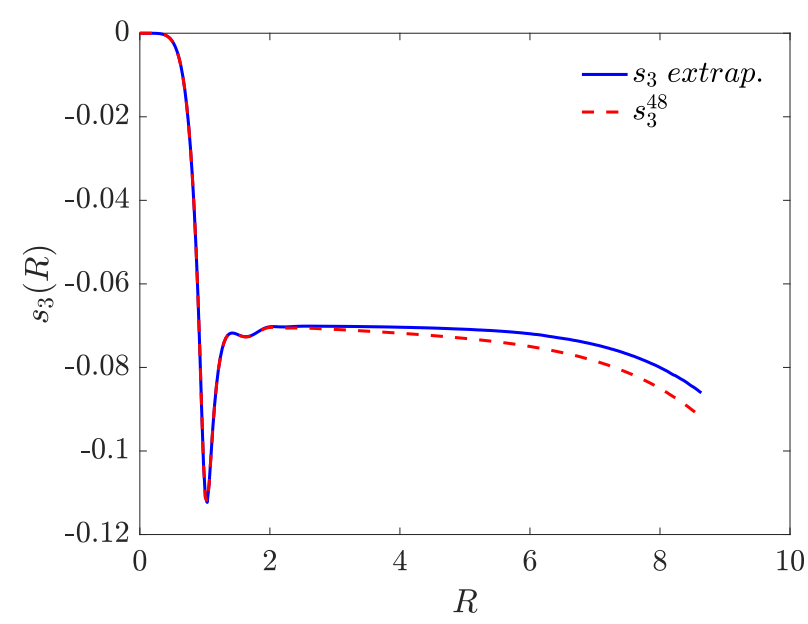

FIG. 5: Three-particle entropy $s_{3}$ for $\rho=0.3, T=1.15$ obtained via the sum of all 48 distributions and extrapolated to the zero noise limit.

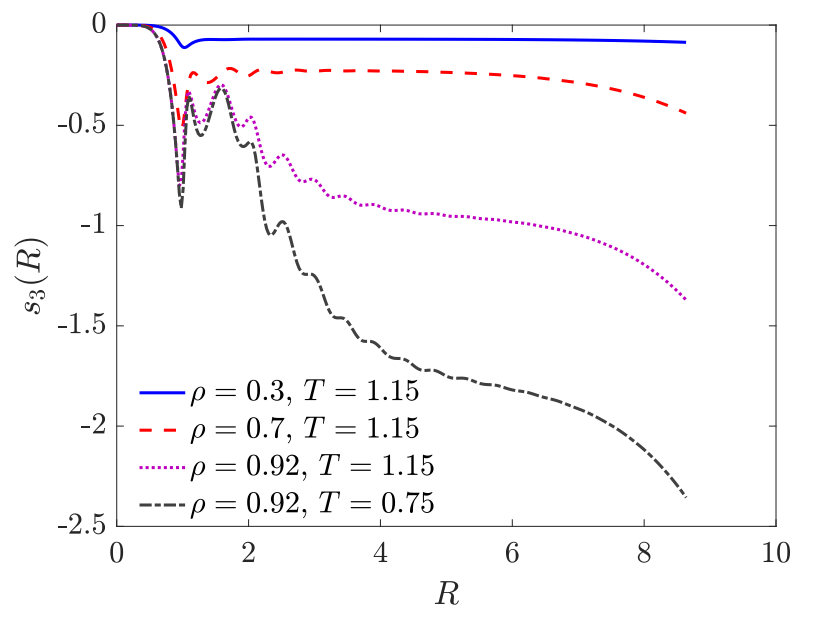

FIG. 6: Three-particle entropy $s_{3}$ for different states, extrapolated to zero noise limit.

the drift; however, this would require much larger computational resources for storing the histograms, whose size scales with the third power of the number of bins per side, and much longer runs, to have an acceptable level of noise.

In Figure 7 we see that the magnitude of the drift is extremely sensitive to the grid size. This phenomenon is particularly severe for high densities. Figure 7 shows the magnitude of the drift for different bin sizes. The convergence is clear as bin size decreases, however, no simple law has been found able to fit the data with accuracy acceptable to have a reliable correction of the drift. We detected at least two different kinds of error dependent on the bin size: the numerical instability, resulting in the drift, and the error caused by the smoothing of the structure of the correlation functions, induced by the histogram approximation. When the bin size is increased, the resulting distribution functions are smoother, and closer to those of a perfect gas. This effect manifests itself in a systematic 


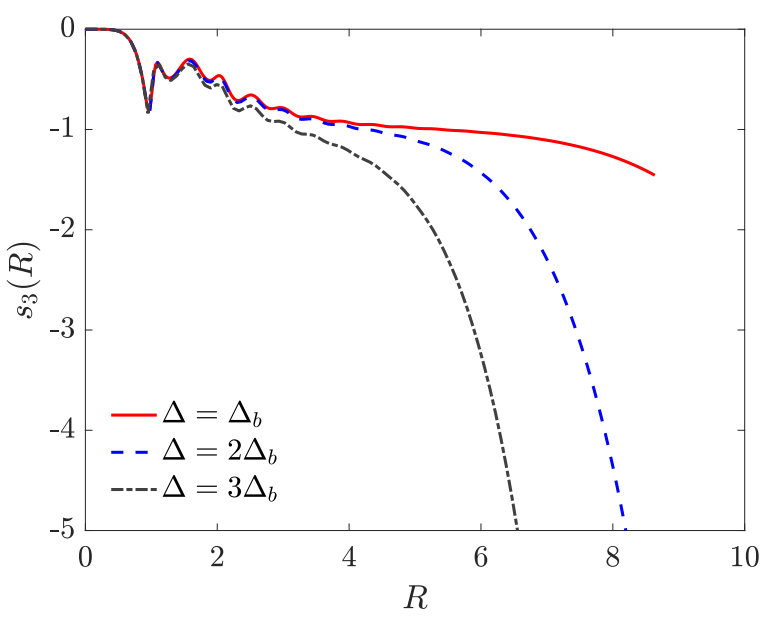

FIG. 7: Grid size effect on the three-particle entropy $(\rho=0.92$, $T=1.15$ ), with $\Delta_{b}=2.88 \times 10^{-2}$ in reduced units.

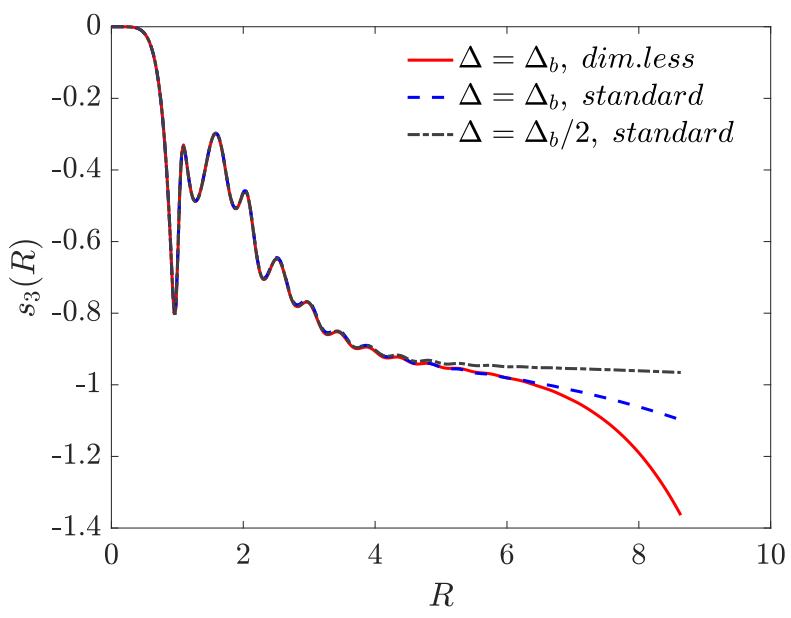

FIG. 8: Grid effect on standard and dimensionless method ( $\rho=0.92, T=1.15) . \Delta_{b}=2.88 \times 10^{-2}$ in reduced units.

under-estimation of the magnitude of $s_{2}$ and $s_{3}$. The second type of error relies on the intimate structure of the correlation functions, and is thus essentially unpredictable without a new theoretical insight into the correlation functions. The interaction between these two effects makes the correction of the drift quite challenging. At this stage the only robust correction of both effects can be achieved by simply choosing a grid fine enough to reduce both errors to a tolerable level. From Figure 8 we see that the drift is generally less severe when the standard method (rather than the dimensionless one) is used, the baseline bin size $\Delta_{b}$ being equal. By exploiting the symmetry of the three-particle function, the histogram of the standard $g^{(3)}$ can be reduced in size, and thus a finer grid can be used. A grid of $\Delta=\Delta_{b} / 2$ is able to reduce the drift to a tolerable level, and $s_{3}$ shows excellent convergence.

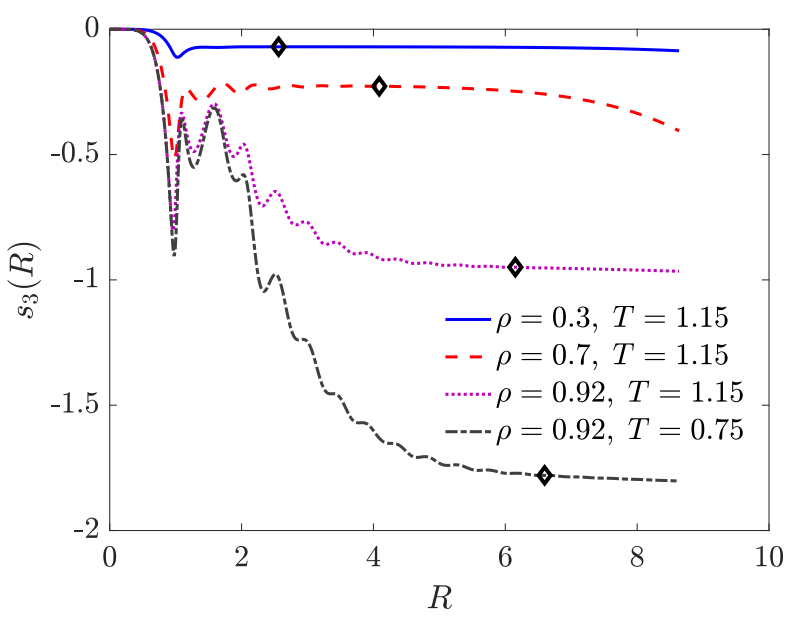

FIG. 9: $s_{3}$ for different state points, and its convergence distance depicted by the diamond points.

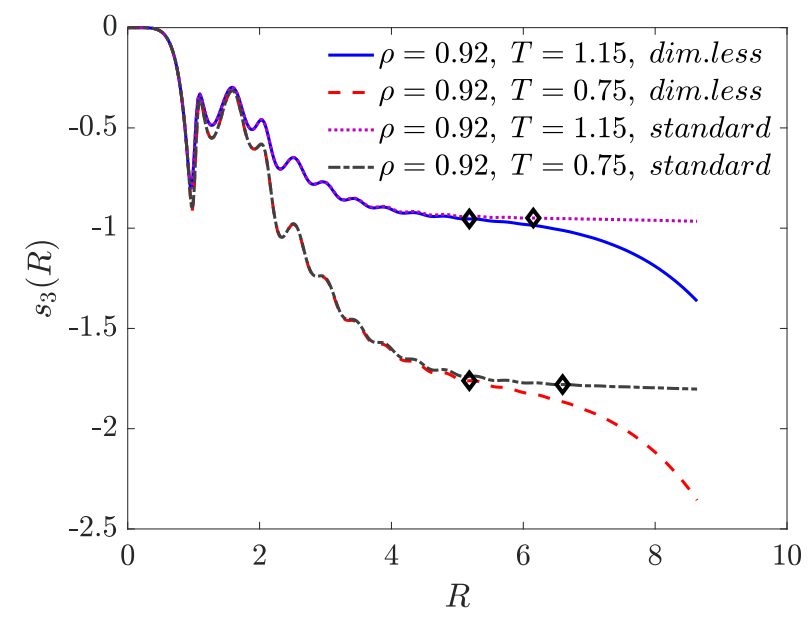

FIG. 10: $s_{3}$ and convergence distance for standard and dimensionless method.

\section{Final estimate}

The presence of numerical instability implies that the convergence of $s_{3}$ can never be reached, thus, a procedure to select its asymptotic value is necessary. The cases of low and intermediate density suggests that when oscillations cease the three-particle entropy is fully converged (Figure 9). At high density, however, the effect of the drift becomes clearly apparent before oscillations become negligible. The best compromise between these two opposite effects was found in the point $R_{\text {conv }}$ in which residual oscillations and numerical instability have the same magnitude. This distance was selected as the separation distance at which the entropy can be considered converged. The region of incomplete convergence $R<R_{\text {conv }}$ is characterized by the presence of stationary points (mimina and maxima), regularly distributed along $R$. When the drift dominates the residual oscillations, at $R>R_{\text {conv }}$, the first 


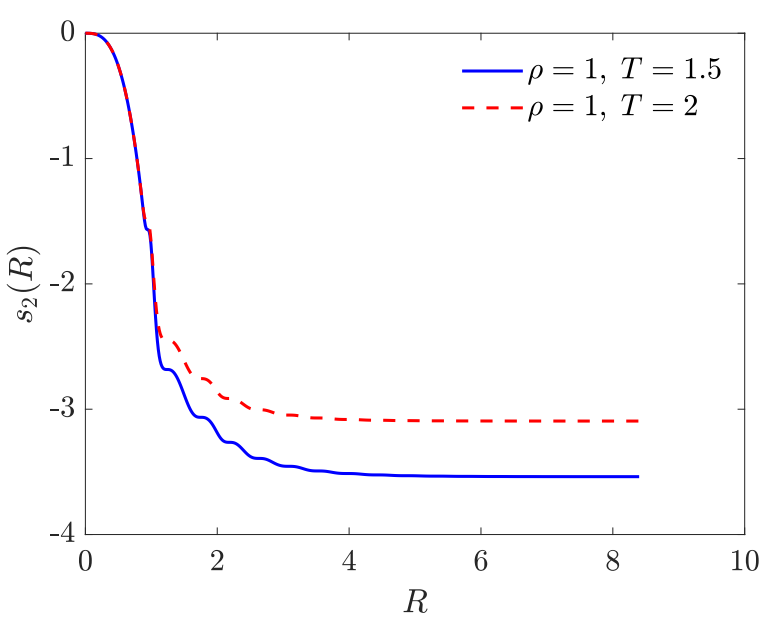

FIG. 11: $s_{2}$ for different state points close to the freezing line.

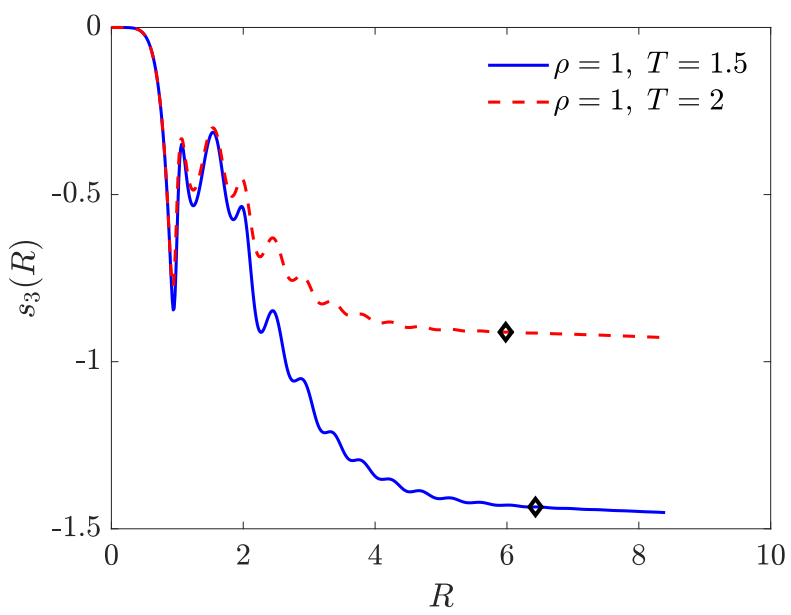

FIG. 12: $s_{3}$ for different state points close to the freezing line $\left(\Delta=\Delta_{b} / 2\right)$.

derivative of $s_{3}$ is, on the other hand, systematically negative. The last stationary point occurring in each $s_{3}$ was thus selected as $R_{\text {conv }}$ and the asymptotic three-particle entropy was set as $s_{3}\left(R_{\text {conv }}\right)$. Figure 10 shows that the numerical instability affecting the dimensionless method with grid size $\Delta_{b}$ leads to a significantly incorrect estimate of $R_{\text {conv }}$ (represented by the diamond symbol), as weak oscillations can still be detected after this distance. The standard method provides a more reliable estimate of $R_{\text {conv }}$, although, quite encouragingly, the relative difference between the entropies was about $0.4 \%$ at $T=1.15$ and $1 \%$ at $T=0.75$.

The state points close to the freezing line were investigated via the standard method, due to their high density. Results are shown in Figures 11 and 12 . For reference, the state points at the solid-liquid phase boundary (coexisting liquid densities) for $(\{T\},\{\rho\})=$ $(\{0.75,1.15,1.5,2\},\{0.915,0.98,1.02,1.07\})$ are taken from Ahmed and Sadus $\frac{12}{2}$.
Table \ provides the final estimate of the two and threeparticle entropy for all states. The error of the estimate is expressed via the standard deviation $\Delta s_{3}$ of the distribution of the values obtained from the extrapolation to the infinite sample limit at $R=R_{\text {conv }}$ (diamond points in Figure 9 12). Comparing the results of WCA with Lennards-Jones particles ${ }^{3}$, we found that the WCA two and three-particle entropy exhibit the same behavior as the Lennard-Jones entropy, the speed of convergence (in terms of separation distance $R$ ) and the oscillatory behavior being essentially the same. The Lennard-Jones entropy has, however, a systematically larger magnitude than the WCA one, in accordance with the attractive component and slower delay of correlations for the Lennard-Jones potential. The two and three-particle entropies were compared with the excess entropy per particle $s_{e x}$

$$
\begin{aligned}
s_{e x}=s-s_{p g} & =\frac{\beta U_{e x}}{N}-\frac{\beta F_{e x}}{N} \\
\frac{\beta U_{e x}}{N} & =\frac{\left\langle E_{p o t}\right\rangle}{N k_{B} T} \\
\frac{\beta F_{e x}}{N} & =\int_{0}^{\rho} \frac{Z-1}{\rho^{\prime}} \mathrm{d} \rho^{\prime}
\end{aligned}
$$

where $U_{e x}$ and $F_{e x}$ are the excess internal energy and the excess Helmholtz free energy. The average potential energy $\left\langle E_{p o t}\right\rangle$, as well as the compressibility factor $Z$, were computed via a WCA equation of state recently found 15 . Table $\amalg$ highlights the magnitude of the two and three-particle entropy with respect to the total excess entropy.

The ratio $s_{3} / s_{2}$ remains quite stable up to intermediate densities ( 0.119 at $\rho=0.3,0.127$ at $\rho=0.7)$ and then increases dramatically when the system is approaching the freezing point $(0.297$ at $\rho=0.92, T=1.15)$. Close to the freezing line, $s_{3}$ is about $45 \%$ of $s_{2}$ at $T=0.75$, and it then decreases with increasing temperature (less than $30 \%$ of the two-particle entropy at $T=2$ ). At high densities the sum of the first two terms, $s_{2}$ and $s_{3}$, exceeds the total excess entropy, a phenomenon already detected for both Lennard-Jones and hard spheres systems 3.8 . This fact entails that the total contribution of higher order terms in the Green's expansion must be positive. We observe that when the freezing line is approached, the overestimation by the three-body contribution to the excess entropy is almost totally nullified by the higher order terms, and the approximation of $s_{e x}$ with $s_{2}$ alone provides a more accurate estimate than $s_{2}+s_{3}$ (Table प). This effect is enhanced by low temperatures, with $s_{2}$ being more than $97 \%$ of the excess entropy at $\rho=0.92$ and $T=0.75$.

We are aware that the data presented in the work of Ahmed and Sadus 12 places the state point $\rho=0.92, T=0.75$ in the region of fluid-solid coexistence. However, the uncertainty about the freezing density and temperature is quite large, as pointed out by the authors themselves when they compare their results with a previous work (see de Kuijper et. $a l^{11}$ ), in which, for instance, the state point $\rho=0.92, T=0.75$ has been found to be still in the fluid regime. The lack of any apparent anomalous behaviour in our computation of the two and three-particle entropy suggests that our results are reliable in the evaluation of the magnitude of such quantities in proximity to that state point. 
TABLE I: Asymptotic values of two and three-particle entropies at different states. $R_{\text {conv }}$ is the convergence distance of $s_{3}$. $\Delta s_{3}$ is the standard deviation of the distribution obtained for the zero noise extrapolation at $R=R_{\text {conv }}$.

\begin{tabular}{cccccc}
\hline \hline$\rho$ & $T$ & $s_{2}$ & $s_{3}$ & $R_{\text {conv }}$ & $\Delta s_{3}$ \\
\hline & & & & & \\
0.3 & 1.15 & -0.5900 & -0.0700 & $2.57 \sigma$ & $1.0 \times 10^{-4}$ \\
0.7 & 1.15 & -1.7880 & -0.2276 & $4.09 \sigma$ & $5.7 \times 10^{-4}$ \\
0.92 & 0.75 & -3.8480 & -1.7798 & $6.42 \sigma$ & $7.1 \times 10^{-4}$ \\
0.92 & 1.15 & -3.2012 & -0.9490 & $6.15 \sigma$ & $5.2 \times 10^{-4}$ \\
1 & 1.5 & -3.5383 & -1.4346 & $6.43 \sigma$ & $4.8 \times 10^{-4}$ \\
1 & 2 & -3.0945 & -0.9115 & $5.98 \sigma$ & $4.4 \times 10^{-4}$ \\
\hline \hline
\end{tabular}

TABLE II: Magnitude of $s_{2}$ and $s_{3}$ with respect to the total excess entropy computed from the equation of state of Mirzaeinia et. al. 15 .

\begin{tabular}{cccccc}
\hline \hline$\rho$ & $T$ & $s_{3} / s_{2}$ & $s_{e x}$ & $s_{2} / s_{e x}$ & $\left(s_{2}+s_{3}\right) / s_{e x}$ \\
\hline & & & & & \\
0.3 & 1.15 & 0.1186 & -0.7118 & 0.8289 & 0.9272 \\
0.7 & 1.15 & 0.1273 & -2.2033 & 0.8115 & 0.9148 \\
0.92 & 0.75 & 0.4625 & -3.9551 & 0.9729 & 1.4229 \\
0.92 & 1.15 & 0.2965 & -3.4823 & 0.9193 & 1.1918 \\
1 & 1.5 & 0.4054 & -3.7255 & 0.9498 & 1.3348 \\
1 & 2 & 0.2946 & -3.4067 & 0.9084 & 1.1759 \\
\hline \hline
\end{tabular}

\section{CONCLUSIONS}

We have presented a new method for the computation of both the three-particle correlation function and the threeparticle entropy for a system of $N$ interacting particles. Although the procedure for the volume correction is well known in the literature, the new dimensionless method involves more straightforward calculations, and it is likely to be more easily generalized to higher order distribution functions, for example to compute the four-particle entropy. The dimensionless method was tested for a WCA fluid at various state points. The numerical instability results from both small number of samples and grid size used to create the histogram for $g^{(3)}$. While the noise effect can be removed with a proper extrapolation, the drift induced by finite bin size is related to the basic structure of the two and three-particle distribution functions, and is thus not easily removed without an extensive investigation of such properties. The dimensionless method is generally more unstable than the traditional one with respect to the grid size, with the computational cost being roughly equal. For low and intermediate densities (up to 0.7), the numerical instability does not compromise the computation of the threeparticle entropy. Higher densities, however, require either a finer grid, or the use of the traditional method. We found that a grid size of $2.88 \times 10^{-2} \sigma$ is fine enough to provide a reliable estimate in most of the cases, with both the standard and dimensionless methods. Coarser grids should be avoided, as they result in greater drift. Since, at this stage, the drift cannot be removed, the selection of a proper estimate for $s_{3}$ requires an adequate criterion. We found that selecting the last stationary point provides a simple and deterministic method, and the best compromise between the error induced by both the numerical instability and the incomplete convergence.
Close to the freezing line, the relative over-estimation of the total excess entropy when the three-body term is included is significantly higher than the underestimation obtained when only the two-body contribution is taken into account.

\section{ACKNOWLEDGEMENTS}

N.C. gratefully acknowledges support from the Australian Research Council under the Future Fellowship scheme (project number FT130100972).

${ }^{1} \mathrm{H}$. S. Green, The Molecular Theory of Fluids (North-Holland, Amsterdam, 1952).

${ }^{2}$ D. Frenkel and B. Smit, Understanding Molecular Simulation (Academic Press, London, 2001).

${ }^{3}$ A. Baranyai and D. J. Evans, "Three-particles contribution to the configurational entropy of simple fluids," Phys. Rev. A 42, 849 (1990).

${ }^{4}$ D. C. Wallace, "On the role of density fluctuations in the entropy of a fluid," J. Chem. Phys. 87, 2282 (1987).

${ }^{5}$ R. E. Nettleton and M. S. Green, "Expression in terms of molecular distribution functions for the entropy density in a infinite system," J. Chem. Phys. 29, 1365 (1958).

${ }^{6}$ H. J. Raveché, "Entropy and molecular correlation functions in open systems.I. Derivation,” J. Chem. Phys. 55, 2242 (1971).

${ }^{7}$ A. Baranyai and D. J. Evans, "Direct entropy calculation for computer simulation of liquids," Phys. Rev. A 40, 3817 (1989).

${ }^{8}$ A. Baranyai and D. J. Evans, "On the entropy of the hard sphere fluid," Zeitschrift für Naturforschung A 46, 27 (1993).

${ }^{9}$ J. D. Weeks, D. Chandler, and H. C. Andersen, "Role of repulsive forces in determining the equilibrium structure of simple liquids," J. Chem. Phys. 54, 5237 (1971)

${ }^{10}$ E. A. Müller and K. E. Gubbins, "Triplet correlation function for hard sphere systems," Mol. Phys. 80, 91 (1993).

${ }^{11}$ A. de Kuijper, J. A. Schouten, and J. P. J. Michels, "The melting line of the Weeks-Chandler-Anderson Lennard-Jones reference system," J. Chem. Phys. 93, 3515 (1990). 
${ }^{12}$ A. Ahmed and R. J. Sadus, "Phase diagram of the Weeks-ChandlerAndersen potential from very low to high temperatures and pressures," Phys. Rev. E 80, 061101 (2009).

${ }^{13}$ M. P. Allen and D. J. Tildesley, Computer Simulation of Liquids (Oxford University Press, Oxford, 1987).

${ }^{14}$ D. Dhabal, A. H. Nguyen, M. Singh, P. Khatua, V. Molinero, S. Bandyopadhyay, and C. Chakravarty, "Excess entropy and crystallization in Stillinger-
Weber and Lennard-Jones fluids," J. Chem. Phys. 143, 164512 (2015).

${ }^{15}$ A. Mirzaeinia, F. Feyzi, and S. M. Hashemianzadeh, "Equation of state and Helmholtz free energy for the atomic system of the repulsive Lennard-Jones particles," J. Chem. Phys. 147, 214503 (2017). 